\title{
The effects of aging on sleep parameters in a healthy, melatonin-competent mouse model
}

This article was published in the following Dove Press journal:

Nature and Science of Sleep

\author{
Jiffin K Paulose \\ Chanung Wang ${ }^{1,2}$ \\ Bruce F O'Hara' \\ Vincent M Cassone' \\ 'Department of Biology, University of \\ Kentucky, Lexington, KY 405I5, USA; \\ ${ }^{2}$ Department of Neurology, Washington \\ University School of Medicine, St. Louis, \\ MO 63II0, USA
}

\begin{abstract}
Background: Sleep disturbances are common maladies associated with human age. Sleep duration is decreased, sleep fragmentation is increased, and the timing of sleep onset and sleep offset is earlier. These disturbances have been associated with several neurodegenerative diseases. Mouse models for human sleep disturbances can be powerful due to the accessibility to neuroscientific and genetic approaches, but these are hampered by the fact that most mouse models employed in sleep research have spontaneous mutations in the biosynthetic pathway(s) regulating the rhythmic production of the pineal hormone melatonin, which has been implicated in human sleep.
\end{abstract}

Purpose and method: The present study employed a non-invasive piezoelectric measure of sleep wake cycles in young, middle-aged and old CBA mice, a strain capable of melatonin biosynthesis, to investigate naturally-occurring changes in sleep and circadian parameters as the result of aging.

Results: The results indicate that young mice sleep less than do middle-aged or aged mice, especially during the night, while the timing of activity onset and acrophase is delayed in aged mice compared to younger mice.

Conclusion: These data point to an effect of aging on the quality and timing of sleep in these mice but also that there are fundamental differences between control of sleep in humans and in laboratory mice.

Keywords: sleep, circadian rhythms, aging, piezoelectric, CBA/J

\section{Introduction}

One of the most common complaints among normal, healthy aged people involves problems with sleep. ${ }^{1,2}$ At the macro level, older people ( $>65$ years) tend to display longer sleep latency, decreased sleep bout length, advanced bed-time, shorter sleep duration, and increased sleep fragmentation. ${ }^{3,4}$ There is also an increase in day-time sleep, or naps, as humans age. ${ }^{5,6}$ These macro level changes in sleep as people age are reflected electrophysiologically in sleep electroencephalography (EEG) in that deeper non-rapid eye movement (nREM) slow wave sleep (SWS) EEG (stages 3 and 4 ) is reduced in many adults aged 75 years or older, ${ }^{7}$ while the numbers of lighter, stage 1 and 2 nREM sleep bouts are increased..$^{8,9}$ Much of the diminution in slow wave activity (SWA) during aging occurs in the prefrontal cortex, where decreases can be as great as $70-80 \%$ relative to younger subjects. ${ }^{8}$

These effects on sleep EEG power are believed to indicate a diminished capacity for the "homeostatic" control of sleep, ${ }^{1}$ according to the two-process model for sleep regulation. ${ }^{10,11}$ In this model, homeostatic sleep regulation reflects an accumulation of "Process S", resulting in the logarithmic growth of "sleep pressure" as
Correspondence: Vincent M Cassone Department of Biology, University of Kentucky, I0I Thomas H. Morgan Building, Lexington, KY 40506-022, USA Tel + I 8592576766

Fax + I 8592571717

Email Vincent.Cassone@uky.edu 
we remain awake. This pressure can only be dissipated through sleep, during which Process S declines exponentially, especially with increased amplitude SWS in Stages 3 and 4 nREM sleep. During aging, it is believed that this homeostatic sleep pressure is reduced, resulting in a decrease in the quality of sleep.

The other process in this two process hypothesis entails a circadian clock, "Process C", located within the hypothalamic suprachiasmatic nucleus (SCN), which determines the timing of wakefulness in the human sleep: wake cycle. ${ }^{12}$ The SCN has been demonstrated to be a master circadian pacemaker for all mammals studied to date. The SCN is a self-sustained circadian oscillator that exhibits circadian rhythms in vivo and in vitro in electrical, metabolic, neurosecretory and gene expression processes. This nucleus is also necessary for circadian rhythms, since surgical destruction of the SCN abolishes all behavioral and physiological rhythms, including those controlling sleep, in animal models ranging from rodents to non-human primates. As one might expect, it would be extremely difficult, if not impossible, to directly measure the effects of aging on SCN function. Even so, Swaab, Hofmann and colleagues have conducted morphometric and immunohistochemical analyses of human SCN obtained post-mortem. ${ }^{13}$ These authors have reported morphometric rhythms in SCN structure and a decrease in the numbers of neurons expressing vasoactive intestinal polypeptide (VIP) in aged post-mortem SCN. ${ }^{14}$

In mammals, the daily and circadian secretion of the indoleamine hormone melatonin by the pineal gland is a direct output of the SCN. ${ }^{15-17}$ Melatonin is secreted during the night and reflects the length of the scotoperiod in all mammals studied thus far, whether they are nocturnal, diurnal or crepuscular ${ }^{15,17}$ and is known to affect sleep: wake cycles in many species of birds and mammals. ${ }^{16}$ Afferents from the SCN are known to innervate subparaventricular hypothalamic neurons, which in turn innervate pre-ganglionic sympathetic neurons in the intermediolateral cell column of the thoracic spinal cord. These neurons innervate the sympathetic ganglionic chain, including the superior cervical ganglion, which innervates the pineal gland, where it secretes the neurotransmitter norepinephrine, stimulating melatonin biosynthesis. Destruction or blockade of any part of this multi-synaptic pathway from the SCN to the pineal gland abolishes melatonin biosynthesis. Thus, one way to assess the activity of the SCN during Process $\mathrm{C}$ is to measure melatonin output.
Aging in humans profoundly affects the amplitude and phase of serum melatonin rhythms. ${ }^{18-21}$ Most studies indicate that the amplitude of serum melatonin decreases during early childhood and continues to decline to approximately $10 \%$ of maximal levels in aged individuals in their $70 \mathrm{~s}$ and $80 \mathrm{~s}$ as compared to younger subjects. ${ }^{18-22}$ In contrast, two studies describe only modest changes in the amplitude of melatonin during aging but instead observe changes in circadian phase, albeit in opposite directions. Ohashi et $\mathrm{al}^{23}$ reported an advance in the melatonin rhythm as subjects age, while Duffy et $\mathrm{al}^{24}$ observed a delay in the circadian phase of the melatonin rhythm. In addition to its role as a biomarker for circadian functions and phase, there is considerable evidence that melatonin also contributes to the quality of sleep if administered at the correct dosages and phases. ${ }^{25}$ These will be discussed in the Discussion below.

The technical and ethical difficulties in studying mechanisms of normal sleep or aging in sleep in human subjects has led many researchers to employ animal models for sleep studies, particularly in laboratory mice. Early EEG studies indicated a decrease in SWS and an increase in wakefulness in healthy, aged $\mathrm{C} 57 \mathrm{BL} / 6 \mathrm{~J}$ and $\mathrm{DBA} / 2 \mathrm{~J}$ mice, ${ }^{26}$ although these authors expressed concerns that the EEG apparatus itself was affecting the quality of sleep. ${ }^{27}$ Later studies by Welsh et $\mathrm{al}^{28}$ reported that old C57BL/6 mice exhibit increased sleep and decreased wakefulness during the active phase of the nocturnal animals at night, and more time awake during the resting, day-time phase. In contrast, Wimmer et $\mathrm{al}^{29}$ did not find decreases in total nREM sleep or increases in wakefulness in aged C57BL/6 mice. Rather, they reported aged mice do not sustain long episodes of wakefulness or nREM sleep, but that there was an increase in total nREM, nREM in the dark and a decrease in overall wakefulness.

A major limitation to the study of sleep with common laboratory strains of mouse is that the most commonly employed strains such as inbred C57BL/6 mice are not capable of producing melatonin due to spontaneous mutations in the rate-limiting enzymes responsible for melatonin biosynthesis, ${ }^{30}$ whereas wild Mus musculus, CBA and $\mathrm{C} 3 \mathrm{H}$ mouse strains are proficient in melatonin biosynthesis. In the case of C57BL/6 inbred strains, a "natural knockdown" in melatonin biosynthesis is due to a truncation in the gene encoding arylalkylamine- $\mathrm{N}$-acetyltransferase (AANAT), ${ }^{31,32}$ while DBA mice exhibit a deficiency in acetylserotonin methyl transferase (ASMT; née hydroxyindole-O-methyl transferase; HIOMT). ${ }^{33}$ Thus, 
understanding any role for endogenous melatonin in sleep regulation in mice is not possible in many strains of mice.

As part of a study on the effects of aging on circadian function of the melatonin-proficient CBA mouse gastrointestinal system, ${ }^{34}$ we also monitored sleep wake patterns employing a piezoelectric, non-invasive sleep monitoring system. $^{35,36}$ This system has been shown to reliably monitor distribution of sleep, sleep quality and transitions to and from rapid eye-movement (REM) sleep, which corresponds favorably with EEG-determined sleep parameters. ${ }^{36}$ The results reveal interesting insights on changes in both sleep quality (Process S) and sleep timing (Process C) during the aging process of melatonin proficient mice.

\section{Methods}

\section{Animals and housing}

Male CBA/J mice (National Institute of Aging, USA) at ages 4 weeks (young, $\mathrm{n}=5$ ), 10 months (middle, $\mathrm{n}=5$ ) and 22 months (old, $n=5$ ) were housed at the University of Kentucky Division of Laboratory Animal Research (UKDLAR). Before piezoelectric sleep recording, animals were housed in $14 \mathrm{hrs}$ : $10 \mathrm{hrs}$ of light:dark (LD 14:10) conditions as per UKDLAR policy. Food (Purina Mouse chow) and water were freely available to the mice throughout the experiment. All procedures were approved by UKDLAR and the Institutional Animal Care and Use Committee. Animal welfare was ensured using USDA guidelines.

\section{Piezoelectric sleep recording}

Activity was recorded using a piezoelectric monitoring system described earlier. ${ }^{35}$ The system uses piezoelectric film to record changes in pressure, the intensity and duration of which are automatically scored by computer algorithms and classified as sleep or wake. Previous use of this system has been validated with $90 \%$ accuracy when compared to EEG. ${ }^{36}$ While validation studies have not been specifically conducted across the entire lifespan of mice or across all mouse strains, the PiezoSleep system has been used by over 100 investigators around the world in many different mouse models of different ages, with no apparent loss of accuracy in sleep-wake classification. Much of this data is unpublished, but see additional references. ${ }^{37-43}$ The primary concern with aged mice might be a reduction in locomotor activity, however, locomotor activity is not a requirement for accurate sleep vs wake determination using the PiezoSleep system, which relies more on breathing regularity during sleep, which is disrupted during even quiet wake by subtle postural adjustments, olfactory sampling (moving the nose), and other small movements that disrupt the regular sleep signature. ${ }^{35,36,42,43}$ Mice were housed individually for two days prior to piezoelectric recording in a light:dark cycle of LD 12:12 and maintained in this photoperiod until the end of recording.

Data collected from the piezoelectric system were binned over 5 mins using a rolling average of the percent sleep, and by individual sleep bout length from which mean bout lengths were calculated. Sleep bouts were defined by any contiguous sleep pattern that remained uninterrupted by arousal periods of more than 30 seconds. In addition, bout length counts were initiated only when a 30-second interval contained greater than $50 \%$ sleep and were terminated when a 30 -second interval contained less than 50\% sleep. Breath rates were averaged for each individual mouse directly from piezoelectric recordings (Figure 1). Mice were returned to normal housing after recording for use in another study. ${ }^{34}$

\section{Statistical analysis}

Measures of sleep parameters included percent Total Sleep over 24 hrs., percent sleep during the day-time hrs., percent sleep in the night-time hrs., time in seconds of the average sleep bout lengths, time in seconds of the average sleep bout length during the day, and time in seconds of the average sleep bout length during the night. Measures of circadian (Process C) sleep regulation were registered in Zeitgeber Time (ZT), where ZT0 corresponds to the time of lights on in an LD 12:12 cycle, and ZT12 corresponds to the time of lights off. These measures included the ZT of activity onset, the ZT of activity offset, and the ZT of peak wake time, which

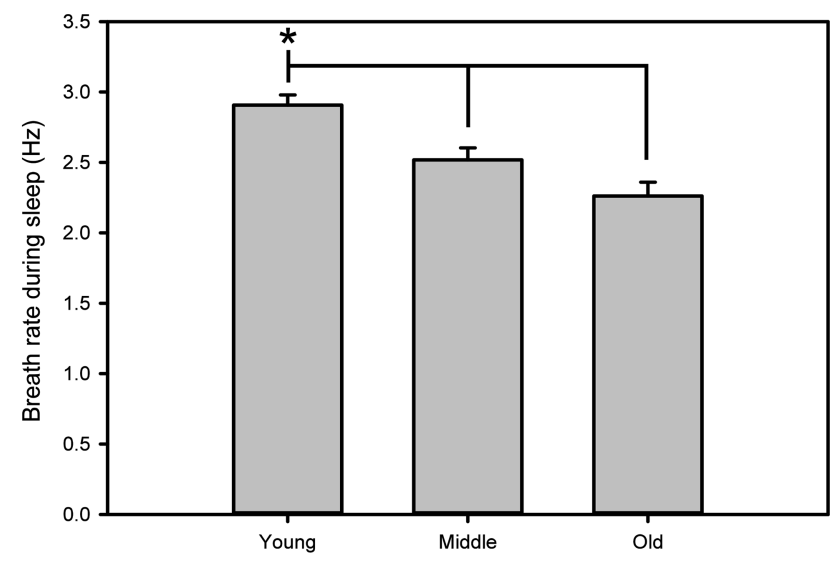

Figure I Breath rate as an indicator of sleep during piezoelectric recording. The piezoelectric recording system uses breath rate to determine active vs sleep states. Here, breath rates during sleep in young mice were significantly different than middle-aged and old mice. Asterisk indicates $p<0.05$. 
were calculated using SleepStats Software (Signal Solutions, LLC) and verified by ActogramJ. ${ }^{44}$

All graphs and statistical tests were performed in Sigmaplot Software (Systat Software, Inc). Percent sleep and sleep parameters were calculated for each individual mouse and then averaged. Comparisons among young, middle-aged, and old mice were tested using Kruskal-Wallis one-way ANOVA on ranks with several non-parametric post-hoc tests. For sleep measures that did not pass Kruskal-Wallis (for total sleep over the course of the day), we applied a Dunn's test, while a Tukey's HSD test was applied to those measures that passed Kruskal-Wallis ANOVA. For circadian parameters, we applied a Holm-Sidak method for multiple comparisons.

\section{Results}

\section{Sleep parameters}

Piezoelectric monitoring of sleep-wake cycles predictably indicated that mice were active predominantly during the dark phase in young, middle-aged and old mice (Figures 2A and 3). Conversely, piezoelectric measures of sleep predominated during the light phase in all three age groups (Figures 2B and 3). In all cases, smaller but significant amounts of activity were detected in all age groups during the light, while smaller but significant sleep activity was observed during the dark.

Measures of sleep from piezoelectric analyses reveal significant age-related changes (Figure 4). Percentage in total sleep/24 hrs. was significantly lower in young vs both old and middle mice (Figure 4A, $p<0.05$ via Dunn's method). Old mice exhibited more time asleep during the day than did young and middle-aged mice (Figure 4B, $p<0.05$ via Tukey's HSD). Young mice also spent less time asleep during the night than did middle-aged mice $(p<0.05$ via Tukey's HSD) (Figure $4 \mathrm{C}$ ) but did not differ from old mice.

Total sleep bout lengths were lower in middle-aged mice than in old mice ( $p<0.05$, Tukey's HSD) but not different from bout length in young mice (Figure 4D). Daytime bout lengths in middle-aged were also lower than bout lengths in old mice ( $p<0.05$, Tukey's HSD), but not different from bout length in young mice (Figure 4E). Nighttime bout length was significantly different between young and old mice $(P<0.05$, Tukey's HSD) (Figure $4 \mathrm{~F})$.
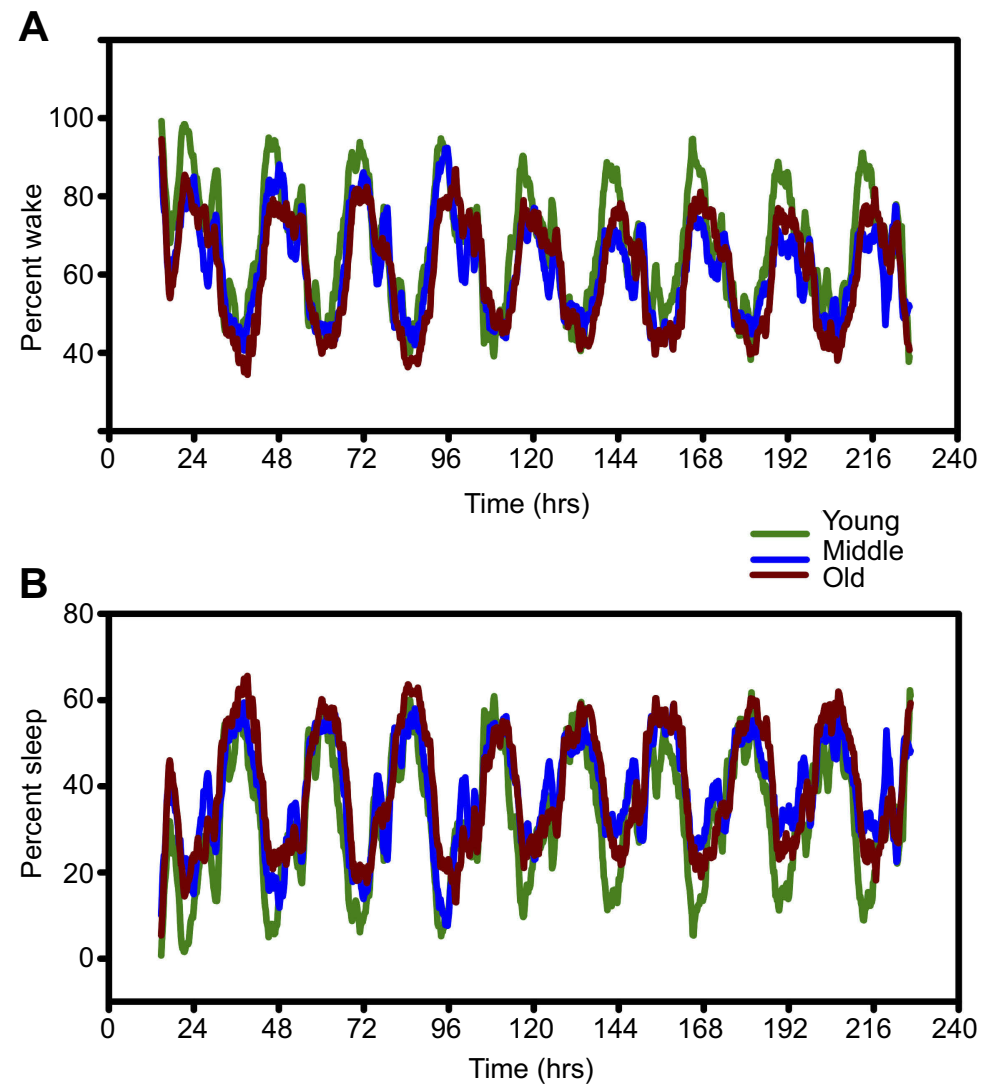

Figure 2 Representative piezoelectric recordings of wake and sleep in young (green lines), middle-aged (blue lines), and old (red lines) mice. Overlaying each trace shows greater differences in wake activity $(\mathbf{A})$ vs sleep $(\mathbf{B})$. 


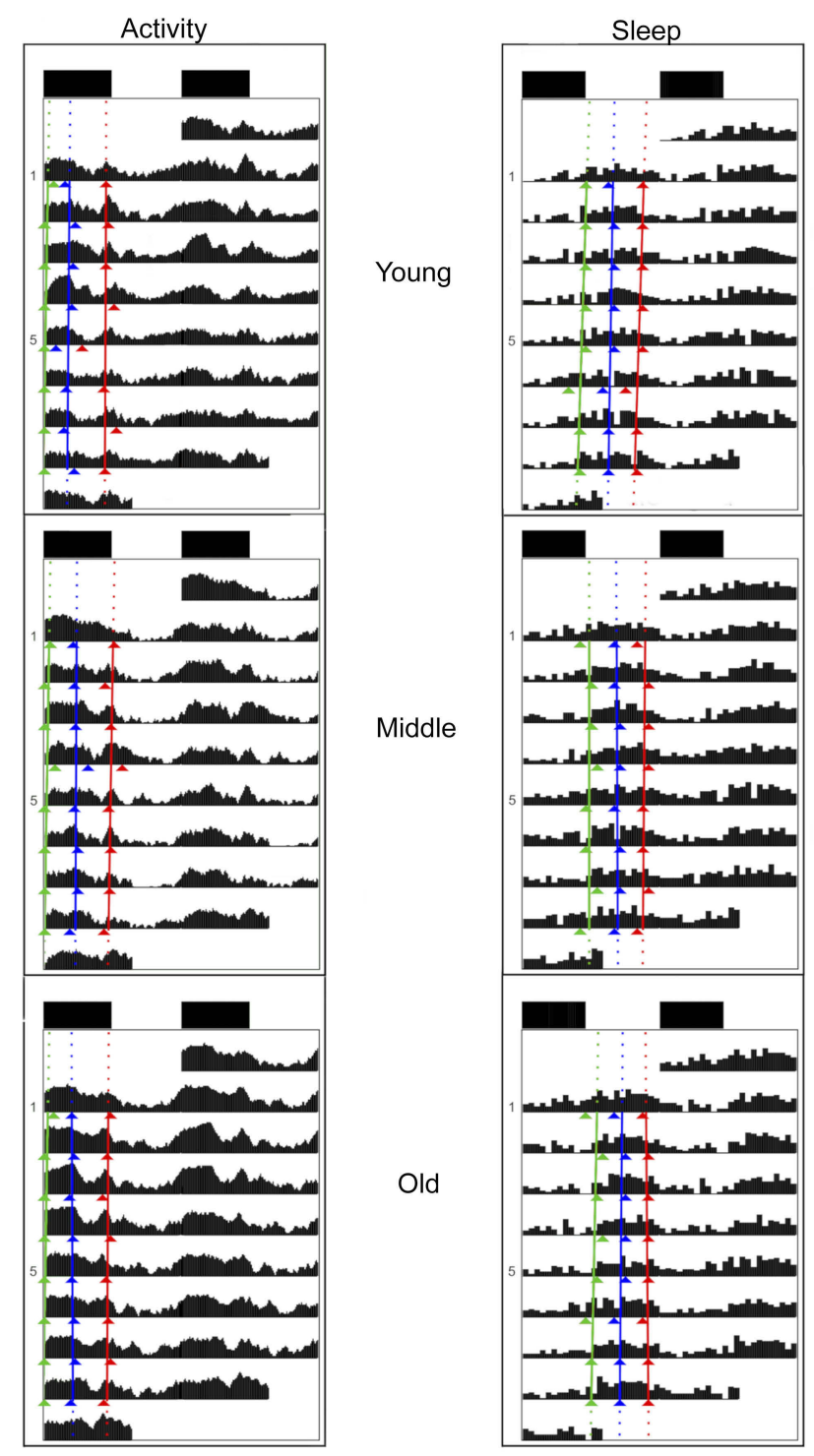

Figure 3 Representative activity (left panels) and sleep (right panels) profiles of young (top pair), middle-aged (middle pair), and old-aged (bottom pair) mice. Within each panel, onset (green lines), peak (blue lines), and offset (red lines) as calculated by ActogramJ are indicated.

\section{Circadian parameters}

Activity onset differed significantly between young and old mice (Figure 5A, $p<0.05$, Holm-Sidak Method). Activity offsets in both young and old mice showed greater variability than middle-aged mice but showed no significant difference between any age group (Figure 5B). Peak phase of activity was significantly delayed in old mice compared to both young and middle-aged mice (Figure 5C, $p<0.05$, HolmSidak Method). Wakefulness in the first three hours after dark onset, which may be interpreted as an indicator of entrainment to the light: dark cycle, was significantly higher in young mice compared to middle-aged and old mice (Figure 5D, $p<0.05$ Tukey's HSD).

\section{Discussion}

The present results are consistent with other studies of healthy mouse models in that both this and previous studies ${ }^{26-28}$ indicated increases in day-time sleep, decreasing night-time wakefulness, and increases in total amounts of sleep as mice age. These data are also similar to at least one aspect of aging human sleep in that both humans and our mice experience increases in sleep during the inactive phase of their sleep-wake cycle; for diurnal humans, there is an increase in day-time sleep, ${ }^{5,6}$ while in mice there is an increase in night-time sleep (Figures 2 and 4). However, they differ from studies of healthy humans in that humans experience decreased sleep bout length and shorter sleep durations, ${ }^{3,4}$ while mice exhibit increased sleep bout length and increased sleep durations (Figure 4). Similarly, circadian parameters differ during aging in humans and mice, since humans experience earlier bed-times, ${ }^{1-4}$ while mice exhibit a phase delay in sleep as they age (Figure 5).

Many studies have identified sleep deficits as contributing factors in degenerative diseases such as Alzheimer's disease (AD), sundown syndrome and other forms of dementia, ${ }^{45-47}$ and many of those factors have been verified through the use of piezoelectric recording. ${ }^{38}$ Sleep disturbances are prevalent and a highly disruptive behavioral symptom in patients with $\mathrm{AD}{ }^{48,49} \mathrm{AD}$ patients experience exacerbated disruptions in the sleep/wake cycle such that they experience increases in sleepiness as the disease progresses and an inability to sleep through the night, so much so that there may be a day/night reversal in sleep phase in the late stages of the disease. ${ }^{47}$ Additionally, a study has reported that sleep disturbances negatively affect cognitive function and functional impairment in $\mathrm{AD}$ patients. ${ }^{50}$ In addition, $\mathrm{AD}$ has been associated with disruption of circadian rhythmicity, such as increased nocturnal activity, decreased diurnal activity, and core body temperature phase delay and amplitude decrease, which have traditionally been assumed to be downstream of the disease. ${ }^{47,51}$ Although neuronal and synaptic damage by $\mathrm{AD}$ pathology progression in the SCN increase the dysfunction of cellular circadian rhythms, which affects sleep disturbance in $\mathrm{AD},{ }^{52}$ it is not clear whether age-related circadian disruption predisposes individuals to the disease, or the disease induces the circadian dysfunction. ${ }^{47}$

Congruently, there is an association of melatonin biosynthesis and secretion with Alzheimer's dementia. ${ }^{47}$ Levels of melatonin in blood and cerebrospinal fluid in Alzheimer's patients are lower than in age-matched healthy subjects. ${ }^{53,54}$ and there is evidence that administration of exogenous melatonin to Alzheimer's patients ameliorates cognitive deficits associated with the disease in living patients as well as decreasing tau deposits in post- 

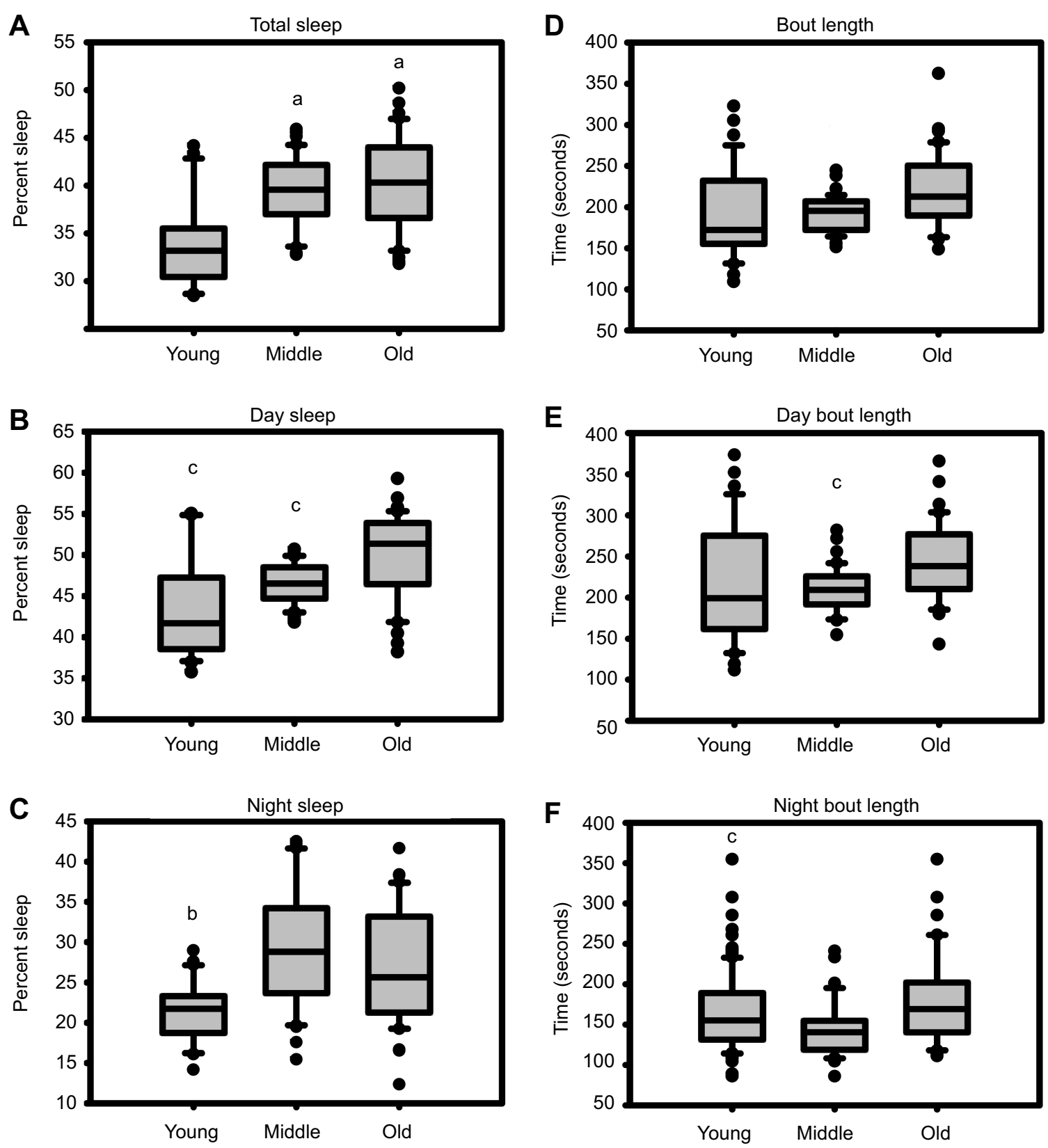

Figure 4 Effects of age on measures of sleep. (A) The percentage of time asleep is lower in young mice than in either middle-aged or old mice. (B) The percentage of time asleep during the day is greater in old mice than in either young or middle-aged mice. (C) The percentage of time asleep during the night is lower in young mice than in either middle-aged or old mice. (D) Average sleep bout length is highly variable. (E, F) Similarly, day-time and night-time sleep bout length is highly variable. (a) indicates significance vs young, (b) indicates significance vs middle-aged, and (c) indicates significance vs old mice.

mortem Alzheimer's brain tissue. ${ }^{55,56}$ It is not clear if the progression of Alzheimer's symptoms affects the circadian secretion of melatonin or whether greater decreases in melatonin levels in aged patients contributes to the progression of the disease. ${ }^{47}$ Properly timed administration of low dosages of melatonin decreases sleep latency and decreases sleep fragmentation in both young adult ${ }^{57}$ and aged $^{58}$ patients. Furthermore, since quality of sleep contributes to the etiology of $\mathrm{AD},{ }^{41}$ it is likely that proper secretion of melatonin is an important factor for the progression of normal aging as well as the diseased state.

Thus, understanding the effects of age on the sleep patterns and other biological processes ${ }^{34}$ in a mouse strain that is capable of synthesizing melatonin may provide a more accurate window into the aging process. Clearly, the fact that we see consistent changes in both sleep and circadian parameters of sleep in our aging mice is an encouraging sign that this may be so. However, we 
Activity onset

A

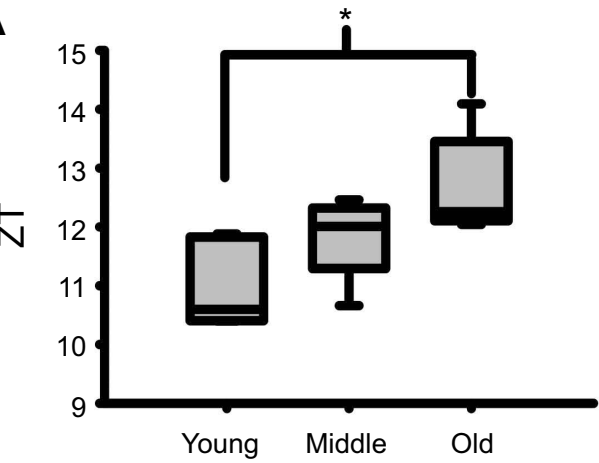

C

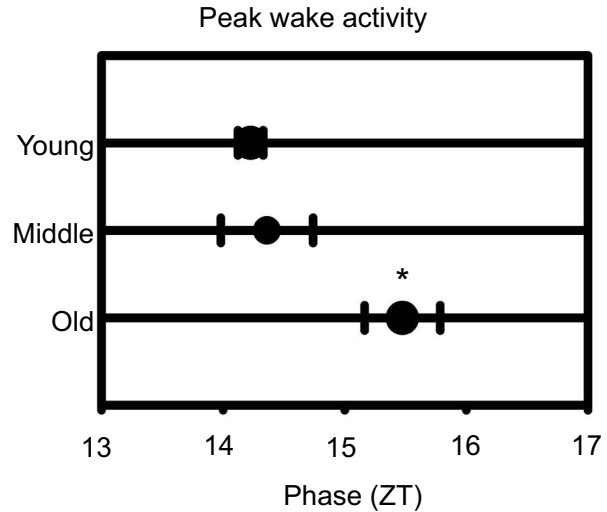

Activity offset

B

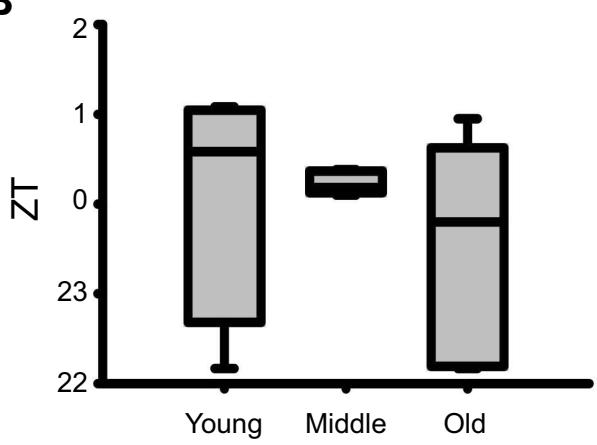

D

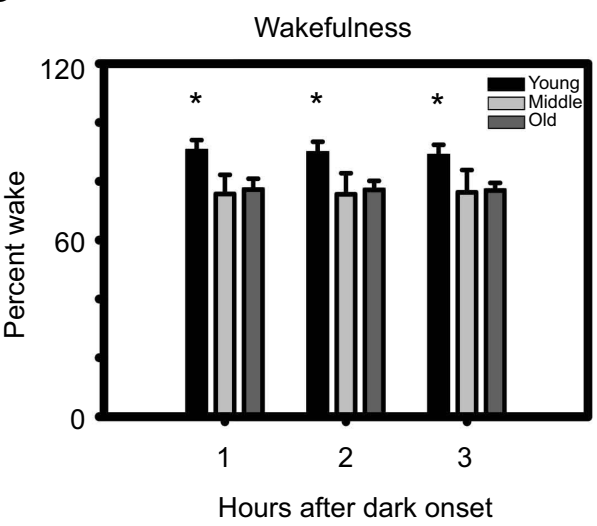

Figure 5 Effects of age on circadian parameters of sleep. (A) Aged mice exhibit a delayed activity onset. (B) No effect of age can be discerned in activity offset. (C) Aged mice exhibit a phase-delayed peak wake activity. (D) Young mice were more wakeful compared to middle-aged and old mice at one, two, and three hours after dark onset. Asterisks indicate $p<0.05$.

recognize that there are fundamental differences between mice and humans in sleep regulation and the effects of age on sleep regulation that raise questions concerning the limitations of mouse models for human health and disease. These may derive from the simple fact that mice are nocturnal, while humans are diurnal, and the fact that melatonin induces sleep in humans ${ }^{25}$ and other diurnal species, while it activates nocturnal rodent locomotor behavior. ${ }^{16}$

\section{Ethical disclosure}

BFO is a co-founder and co-owner of Signal Solutions, LLC (Lexington, KY) that manufactures and sells the PiezoSleep system used in this study.

\section{Disclosure}

Dr Bruce F O'Hara is a co-founder and co-owner of Signal Solutions LLC, that manufatures the PiezoSleep technology used in this study. However, his role in this study was as a Professor of Biology, and nothing in this work has significant financial ramifications for Signal Solutions
LLC, and thus the conflict of interest is very low to nonexistent in this case. The other authors report no conflicts of interest in this work.

\section{References}

1. Mander BA, Winer JR, Walker MP. Sleep and human aging. Neuron. 2017;94(1):19-36. doi:10.1016/j.neuron.2017.02.004

2. Zhong -H-H, Yu B, Luo D, et al. Roles of aging in sleep. Neurosci Biobehav Rev. 2019;98:177-184. doi:10.1016/j.neubiorev.2019.01.013

3. Klerman EB, Dijk D-J. Age-related reduction in the maximal capacity for sleep-implications for insomnia. Curr Biol. 2008;18(15):11181123. doi:10.1016/j.cub.2008.06.047

4. Kim JH, Duffy JF. Circadian rhythm sleep-wake disorders in older adults. Sleep Med Clin. 2018;13(1):39-50. doi:10.1016/j.jsmc.2017.09.004

5. Dijk D-J, Groeger JA, Stanley N, Deacon S. Age-related reduction in daytime sleep propensity and nocturnal slow wave sleep. Sleep. 2010;33(2):211-223. doi:10.1093/sleep/33.2.211.

6. Foley DJ, Vitiello MV, Bliwise DL, Ancoli-Israel S, Monjan AA, Walsh JK. Frequent napping is associated with excessive daytime sleepiness, depression, pain, and nocturia in older adults: findings from the National Sleep Foundation "2003 Sleep in America" poll. Am J Geriatr Psychiatry. 2007;15(4):344-350. doi:10.1097/01. JGP.0000249385.50101.67

7. Ohayon MM, Carskadon MA, Guilleminault C, Vitiello MV. Meta-analysis of quantitative sleep parameters from childhood to old age in healthy individuals: developing normative sleep-values across the human lifespan. Sleep. 2004. 27(7):1255-1273. doi:10.1093/sleep/27.7.1255. 
8. Carrier J, Land S, Buysse DJ, Kupfer DJ, Monk TH. The effects of age and gender on sleep EEG power spectral density in the middle years of life (ages 20-60 years old). Psychophysiology. 2001;38 (2):232-242. doi:10.1111/psyp.2001.38.issue-2.

9. Landolt HP, Dijk DJ, Achermann P, Borbély AA. Effect of age on the sleep EEG: slow-wave activity and spindle frequency activity in young and middle-aged men. Brain Res. 1996. 738(2):205-212. doi:10.1016/s0006-8993(96)00770-6.

10. Daan S, Beersma DG, Borbely AA. Timing of human sleep: recovery process gated by a circadian pacemaker. Am J Physiol Integr Comp Physiol. 1984;246(2):R161-R183. doi:10.1152/ajpregu.1984.246.2.R161

11. Borbély AA, Daan S, Wirz-Justice A, Deboer T. The two-process model of sleep regulation: a reappraisal. J Sleep Res. 2016;25 (2):131-143. doi:10.1111/jsr.12371

12. Moore RY. The suprachiasmatic nucleus and the circadian timing system. In: Gillette, MU. editor. Progress in Molecular Biology and Translational Science. Vol. 119. 2013;1-28. doi: 10.1016/B978-0-12396971-2.00001-4

13. Hofman MA, Zhou J-N, Swaab DF. Suprachiasmatic nucleus of the human brain: an immunocytochemical and morphometric analysis. Anat Rec. 1996;244(4):552-562. doi:10.1002/(SICI)1097-0185 (199604)244:4<552::AID-AR13>3.0.CO;2-O

14. HOFMAN M, SWAAB D. Living by the clock: the circadian pacemaker in older people. Ageing Res Rev. 2006;5(1):33-51. doi:10.1016/j.arr.2005.07.001

15. Cassone VM. Melatonin: time in a bottle. Oxf Rev Reprod Biol. 1990;12:319-367. Available from: http://www.ncbi.nlm.nih.gov/ pubmed/2075003. Accessed April 2, 2019.

16. Cassone VM. Melatonin's role in vertebrate circadian rhythms. Chronobiol Int. 1998;15(5):457-473. Available from: http://www. ncbi.nlm.nih.gov/pubmed/9787936. Accessed June 27, 2013.

17. Klein DC, Coon SL, Roseboom PH, et al. The melatonin rhythmgenerating enzyme: molecular regulation of serotonin N-acetyltransferase in the pineal gland. Recent Prog Horm Res. 1997;52:307-357; discussion 357-8. Available from: http://www.ncbi.nlm.nih.gov/ pubmed/9238858. Accessed June 27, 2013.

18. Nair NP, Hariharasubramanian N, Pilapil C, Isaac I, Thavundayil JX. Plasma melatonin-an index of brain aging in humans? Biol Psychiatry. 1986;21(2):141-150. Available from: http://www.ncbi. nlm.nih.gov/pubmed/3947693. Accessed April 2, 2019.

19. Skene DJ, Vivien-Roels B, Sparks DL, et al. Daily variation in the concentration of melatonin and 5-methoxytryptophol in the human pineal gland: effect of age and Alzheimer's disease. Brain Res. 1990;528(1):170-174. Available from: http://www.ncbi.nlm.nih.gov/ pubmed/2245336. Accessed April 2, 2019.

20. Waldhauser F, Weiszenbacher G, Tatzer E, et al. Alterations in nocturnal serum melatonin levels in humans with growth and aging. $J$ Clin Endocrinol Metab. 1988;66(3):648-652. doi:10.1210/jcem-66-3-648

21. Reiter RJ. Pineal function during aging: attenuation of the melatonin rhythm and its neurobiological consequences. Acta Neurobiol Exp (Wars). 1994;54 Suppl:31-39. Available from: http://www.ncbi.nlm. nih.gov/pubmed/7801790. Accessed April 2, 2019.

22. Reiter RJ. The pineal gland and melatonin in relation to aging: A summary of the theories and of the data. Exp Gerontol. 1995;30(34):199-212. doi:10.1016/0531-5565(94)00045-5

23. Ohashi $\mathrm{Y}$, Okamoto N, Uchida K, Iyo M, Mori N, Morita Y. Differential pattern of the circadian rhythm of serum melatonin in young and elderly healthy subjects. Biol Signals. 1997;6(4-6):301306. doi:10.1159/000109141.

24. Duffy JF, Zeitzer JM, Rimmer DW, Klerman EB, Dijk D-J, Czeisler CA. Peak of circadian melatonin rhythm occurs later within the sleep of older subjects. Am J Physiol Metab. 2002;282(2):E297-E303. doi:10.1152/ajpendo.00268.2001

25. Touitou Y. Human aging and melatonin. Clinical relevance. Exp Gerontol. 2001;36(7):1083-1100. Available from: http://www.ncbi. nlm.nih.gov/pubmed/11404053. Accessed April 2, 2019.
26. Eleftheriou BE, Zolovick AJ, Elias MF. Electroencephalographic changes with age in male mice. Gerontologia. 1975;21(1):21-30. Available from: http://www.ncbi.nlm.nih.gov/pubmed/166900. Accessed November 17, 2017.

27. Elias MF, Elias PK, Zolovick AJ, Eleftheriou BE. Some methodological problems in age comparisons of EEG sleep patterns for C57BL/ 6J mice. Exp Aging Res. 1975;1(1):107-119. doi:10.1080/ 03610737508257952

28. Welsh DK, Richardson GS, Dement WC. Effect of age on the circadian pattern of sleep and wakefulness in the mouse. $J$ Gerontol. 1986;41(5):579-586. Available from: http://www.ncbi. nlm.nih.gov/pubmed/3745812. Accessed December 21, 2017.

29. Wimmer ME, Rising J, Galante RJ, Wyner A, Pack AI, Abel T. Aging in mice reduces the ability to sustain sleep/wake states. Norris CM, ed. PLoS One. 2013:8(12):e81880. doi:10.1371/journal.pone.0081880.

30. Ebihara S, Marks T, Hudson DJ, Menaker M. Genetic control of melatonin synthesis in the pineal gland of the mouse. Science. 1986;231(4737):491-493. doi:10.1126/SCIENCE.3941912

31. Roseboom PH, Namboodiri MA, Zimonjic DB, et al. Natural melatonin "knockdown" in C57BL/6J mice: rare mechanism truncates serotonin N-acetyltransferase. Brain Res Mol Brain Res. 1998;63 (1):189-197. Available from: http://www.ncbi.nlm.nih.gov/pubmed/ 9838107. Accessed February 15, 2019.

32. von Gall C, Lewy A, Schomerus C, et al. Transcription factor dynamics and neuroendocrine signalling in the mouse pineal gland: a comparative analysis of melatonin-deficient C57BL mice and melatonin-proficient C3H mice. Eur J Neurosci. 2000;12(3):964-972. Available from: http://www.ncbi.nlm.nih.gov/pubmed/10762326. Accessed April 2, 2019.

33. Slominski A, Pisarchik A, Semak I, Sweatman T, Wortsman J. Characterization of the serotoninergic system in the C57BL/6 mouse skin. Eur J Biochem. 2003. 270(16):3335-3344. doi:10.1046/j.14321033.2003.03708.x.

34. Paulose JK, Cassone CV, Cassone VM. Aging, melatonin biosynthesis, and circadian clockworks in the gastrointestinal system of the laboratory mouse. Physiol Genomics. 2019;51(1):1-9. doi:10.1152/ physiolgenomics.00095.2018

35. Donohue KD, Medonza DC, Crane ER, O'Hara BF. Assessment of a non-invasive high-throughput classifier for behaviours associated with sleep and wake in mice. Biomed Eng Online. 2008;7(1):14. doi:10.1186/1475-925X-7-14

36. Mang GM, Nicod J, Emmenegger Y, Donohue KD, O'Hara BF, Franken P. Evaluation of a piezoelectric system as an alternative to electroencephalogram/electromyogram recordings in mouse sleep studies. Sleep. 2014;37(8):1383-1392. doi:10.5665/sleep.3936

37. Philip VM, Sokoloff G, Ackert-Bicknell CL, et al. Genetic analysis in the collaborative cross breeding population. Genome Res. 2011;21 (8):1223-1238. doi:10.1101/gr.113886.110

38. Duncan MJ, Smith JT, Franklin KM, et al. Effects of aging and genotype on circadian rhythms, sleep, and clock gene expression in APPxPS1 knock-in mice, a model for Alzheimer's disease. Exp Neurol. 2012;236(2):249-258. doi:10.1016/j.expneurol.2012.05.011

39. Rowe RK, Harrison JL, O'Hara BF, Lifshitz J. Diffuse brain injury does not affect chronic sleep patterns in the mouse. Brain Inj. 2014;28(4):504-510. doi:10.3109/02699052.2014.888768

40. Rowe RK, Striz M, Bachstetter AD, et al. Diffuse brain injury induces acute post-traumatic sleep. de Castro F, ed. PLoS One. 2014;9(1):e82507. doi:10.1371/journal.pone.0082507

41. Sethi M, Joshi SSS, Webb RLL, et al. Increased fragmentation of sleep-wake cycles in the 5XFAD mouse model of Alzheimer's disease. Neuroscience. 2015;290:80-89. doi:10.1016/j.neuroscience. 2015.01.035

42. Yaghouby F, O'Hara BF, Sunderam S. Unsupervised estimation of mouse sleep scores and dynamics using a graphical model of electrophysiological measurements. Int $J$ Neural Syst. 2016;26 (04):1650017. doi:10.1142/S0129065716500179 
43. Yaghouby F, Donohue KD, O’Hara BF, Sunderam S. Noninvasive dissection of mouse sleep using a piezoelectric motion sensor. $J$ Neurosci Methods. 2016;259:90-100. doi:10.1016/j.jneumeth.2015.11.004

44. Rasband WS Image J. U S Natl Institutes Heal Bethesda, Maryland, USA; 1997-2014. Available from: http://imagej.nih.gov/ij/. Accessed January 14, 2019.

45. Vitiello MV, Prinz PN. Alzheimer's disease. Sleep and sleep/wake patterns. Clin Geriatr Med. 1989;5(2):289-299. Available from: http:// www.ncbi.nlm.nih.gov/pubmed/2665912. Accessed April 2, 2019.

46. Bubu OM, Brannick M, Mortimer J, et al. Sleep, cognitive impairment, and Alzheimer's disease: a systematic review and meta-analysis. Sleep. 2017;40(1). doi:10.1093/sleep/zsw032

47. Bedrosian TA, Nelson RJ. Pro: alzheimer's disease and circadian dysfunction: chicken or egg? Alzheimer's Res Ther. 2012;4(4). doi:10.1186/alzrt128

48. Moran M, Lynch CA, Walsh C, Coen R, Coakley D, Lawlor BA. Sleep disturbance in mild to moderate Alzheimer's disease. Sleep Med. 2005;6(4):347-352. doi:10.1016/j.sleep.2004.12.005

49. Ju Y-ES, Lucey BP, Holtzman DM. Sleep and Alzheimer disease pathology-a bidirectional relationship. Nat Rev Neurol. 2014;10 (2):115-119. doi:10.1038/nrneurol.2013.269

50. Ju Y-ES, McLeland JS, Toedebusch CD, et al. Sleep quality and preclinical Alzheimer disease. JAMA Neurol. 2013;70(5):587. doi:10.1001/jamaneurol.2013.2334

51. Witting W, Kwa IH, Eikelenboom P, Mirmiran M, Swaab DF. Alterations in the circadian rest-activity rhythm in aging and Alzheimer's disease. Biol Psychiatry. 1990;27(6):563-572. Available from: http://www.ncbi.nlm.nih.gov/pubmed/2322616. Accessed April 30,2019 .
52. Harper DG, Stopa EG, Kuo-Leblanc V, et al. Dorsomedial SCN neuronal subpopulations subserve different functions in human dementia. Brain. 2008;131(Pt 6):1609-1617. doi:10.1093/brain/ awn049

53. Liu R-Y, Zhou J-N, van Heerikhuize J, Hofman MA, Swaab DF. Decreased melatonin levels in postmortem cerebrospinal fluid in relation to aging, Alzheimer's disease, and apolipoprotein E- $\varepsilon 4 / 4$ genotype $^{1} . J$ Clin Endocrinol Metab. 1999;84(1):323-327. doi:10.1210/jcem.84. 1.5394

54. Jennum P, Lykke Mortensen E, Gammeltoft S, et al. Melatonin and cortisol profiles in late midlife and their association with age-related changes in cognition. Nat Sci Sleep. 2016;47. doi:10.2147/NSS. S75946

55. Balmik AA, Chinnathambi S. Multi-faceted role of melatonin in neuroprotection and amelioration of Tau aggregates in Alzheimer's disease. J Alzheimer's Dis. 2018;62(4):1481-1493. doi:10.3233/JAD170900

56. Vincent B. Protective roles of melatonin against the amyloid-dependent development of Alzheimer's disease: A critical review. Pharmacol Res. 2018;134:223-237. doi:10.1016/j.phrs.2018.06.011

57. Zhdanova IV, Wurtman RJ. Efficacy of melatonin as a sleep-promoting agent. J Biol Rhythms. 1997;12(6):644-650. doi:10.1177/0748730497 01200620

58. Zhdanova IV, Wurtman RJ, Regan MM, Taylor JA, Shi JP, Leclair OU. Melatonin treatment for age-related insomnia. J Clin Endocrinol Metab. 2001;86(10):4727-4730. doi:10.1210/jcem.86.10.7901

\section{Publish your work in this journal}

Nature and Science of Sleep is an international, peer-reviewed, open access journal covering all aspects of sleep science and sleep medicine, including the neurophysiology and functions of sleep, the genetics of sleep, sleep and society, biological rhythms, dreaming, sleep disorders and therapy, and strategies to optimize healthy sleep
The manuscript management system is completely online and includes a very quick and fair peer-review system, which is all easy to use. Visit http://www.dovepress.com/testimonials.php to read real quotes from published authors. 